\title{
Tandem Oxidation Processes for the Preparation of Functionalised Cyclopropanes
}

\author{
Magalie F. Oswald, Steven A. Raw, Richard J. K. Taylor* \\ Department of Chemistry, University of York, Heslington, York, YO10 5DD, UK \\ rjkt1@york.ac.uk
}

\section{Supplementary Infomation}

Compounds $3 \mathbf{a},{ }^{4 \mathrm{c}} \mathbf{3 b},{ }^{4 \mathrm{a}} \mathbf{3} \mathbf{c},{ }^{4 \mathrm{~b}} \mathbf{3 g},{ }^{4 \mathrm{~d}} \mathbf{3 h},{ }^{4 \mathrm{e}} \mathbf{3 i},{ }^{4 \mathrm{f}} \mathbf{3 o},{ }^{4 \mathrm{~g}} \mathbf{3} \mathbf{p},{ }^{4 \mathrm{~h}} \mathbf{3} \mathbf{r}^{4 \mathrm{i}}$ and $\mathbf{3} \mathbf{t}^{9}$ are known compounds and the data correspond with those reported in the literature.

至

To a solution of allylic alcohol $\mathbf{1 a}(36 \mathrm{mg}, 0.50 \mathrm{mmol})$ in dichloromethane $(5 \mathrm{~mL})$ was added powdered $4 \AA$ molecular sieves $(0.50 \mathrm{~g})$, (benzoylmethylene)dimethylsulfurane $\mathbf{2 b}$ (108 $\mathrm{mg}$, $0.60 \mathrm{mmol})$ and manganese dioxide $(435 \mathrm{mg}, 5.0 \mathrm{mmol})$. The mixture was heated at reflux for 2 hours then cooled to room temperature. The crude mixture was then filtered through celite ${ }^{\circledR}$ and the residue washed with dichloromethane $(50 \mathrm{~mL})$ giving a pale yellow solution. After removal of the solvent, the residue was purified by flash column chromatography $(19: 1$ to $4: 1 \mathrm{PE}:$ EtOAc) to give the title compound 3d (50 mg, 53\%) as a colorless oil, $4.2: 1$ trans: cis by ${ }^{1} \mathrm{H}$ NMR integration. $v_{\max }$ (film) 2935, 1712, 1665, 1596, 1449, 1379, 1305, 1225 $\mathrm{cm}^{-1}$; ${ }^{1} \mathrm{H}$ NMR (400 MHz, $\left.\mathrm{CDCl}_{3}\right)$ trans: $\delta 1.26(3 \mathrm{H}, \mathrm{s}, \mathrm{Me}), 1.59(1 \mathrm{H}, \mathrm{dd}, J 4.9 \mathrm{~Hz}, J 8.2$ Hz), $1.91(1 \mathrm{H}, \mathrm{dd}, J 4.9 \mathrm{~Hz}, J 7.0 \mathrm{~Hz}), 3.20(1 \mathrm{H}, \mathrm{dd}, J 8.2 \mathrm{~Hz}, J 7.0 \mathrm{~Hz}), 7.47$ (2 H, dd (app. t), J 7.6 Hz, arom.), 7.58 (1 H, dd (app. t), J 7.6 Hz, arom.), 7.90 (1 H, d, J 7.6 Hz, arom.), $9.09(1 \mathrm{H}, \mathrm{s}, \mathrm{CHO})$ cis: $\delta 1.44(3 \mathrm{H}, \mathrm{s}, \mathrm{Me}), 1.54(1 \mathrm{H}, \mathrm{dd}, J 4.9 \mathrm{~Hz}, J 7.6 \mathrm{~Hz}), 2.39(1 \mathrm{H}, \mathrm{dd}, J$ $4.9 \mathrm{~Hz}, J 7.0 \mathrm{~Hz}), 3.01(1 \mathrm{H}, \mathrm{dd}, J 7.6 \mathrm{~Hz}, J 7.0 \mathrm{~Hz}), 7.47$ (2 H, dd (app. t), $J 7.6 \mathrm{~Hz}$, arom.), 7.58 (1 H, dd (app. t), J 7.6 Hz, arom.), 7.93 (1 H, d, J 7.6 Hz, arom.), $9.11(1 \mathrm{H}, \mathrm{s}, \mathrm{CHO})$; ${ }^{13} \mathrm{C}$ NMR $\left(100 \mathrm{MHz}, \mathrm{CDCl}_{3}\right)$ trans: $\delta 10.3\left(\mathrm{CH}_{3}\right), 18.6\left(\mathrm{CH}_{2}\right), 29.9(\mathrm{CH}), 37.4(\mathrm{C}), 128.3$ $(\mathrm{CH}), 128.9(\mathrm{CH}), 133.6(\mathrm{CH}), 137.8(\mathrm{C}), 194.9(\mathrm{C}), 200.0(\mathrm{C})$ cis: $\delta 17.5\left(\mathrm{CH}_{3}\right), 21.2\left(\mathrm{CH}_{2}\right)$, $35.0(\mathrm{CH}), 37.8(\mathrm{C}), 128.4(\mathrm{CH}), 128.9(\mathrm{CH}), 133.7(\mathrm{CH}), 137.2(\mathrm{C}), 195.6(\mathrm{C}), 200.4(\mathrm{C})$; $m / z(\mathrm{CI}) 189\left(\mathrm{MH}^{+}\right)$[HRMS $(\mathrm{CI})\left[\mathrm{MH}^{+}\right] \mathrm{C}_{12} \mathrm{H}_{13} \mathrm{O}_{2}$ requires 189.0916. Found: $189.0912(2.1 \mathrm{ppm}$ error).

$\mathrm{EtO}_{2} \mathrm{C}_{2}$

To a solution of allylic alcohol $1 \mathbf{c}(101 \mathrm{mg}, 0.50 \mathrm{mmol})$ in dichloromethane $(5 \mathrm{~mL})$ was added powdered $4 \AA$ molecular sieves $(0.50 \mathrm{~g})$, (carbethoxymethylene)dimethylsulfurane $\mathbf{2 a}(90 \mathrm{mg}$, $0.60 \mathrm{mmol}$ ) and manganese dioxide $(430 \mathrm{mg}, 4.9 \mathrm{mmol})$. The mixture was heated at reflux for 18 hours, cooled to room temperature and stirred for a further 8 hours. The crude mixture was then filtered through celite ${ }^{\circledR}$ and the residue washed with dichloromethane $(50 \mathrm{~mL})$ giving a pale yellow solution. After removal of the solvent, the residue was purified by flash column chromatography (100\% petrolum ether) to give the title compound $3 \mathbf{e}(100 \mathrm{mg}, 59 \%)$ as a colorless oil, $\sim 3.5: 1$ trans: cis by ${ }^{1} \mathrm{H}$ NMR integration. $v_{\max }$ (film) 779, 839, 1097, 1176, 1257 , 
1704, 1732, 2856, 2931, $2953 \mathrm{~cm}^{-1} ;{ }^{1} \mathrm{H}$ NMR $\left(270 \mathrm{MHz}, \mathrm{CDCl}_{3}\right)$ trans: $\delta 0.02(3 \mathrm{H}, \mathrm{s}, \mathrm{SiMe})$, $0.05(3 \mathrm{H}, \mathrm{s}, \mathrm{SiMe}) ; 0.84\left(9 \mathrm{H}, \mathrm{s}, \mathrm{SiCMe}_{3}\right), 1.23-1.28\left(3 \mathrm{H}, \mathrm{m}, \mathrm{CO}_{2} \mathrm{CH}_{2} \mathrm{CH}_{3}\right), 1.45(1 \mathrm{H}, \mathrm{dd}, J$ $4.5 \mathrm{~Hz}, J 8.5 \mathrm{~Hz}), 1.53(1 \mathrm{H}$, dd, $J 4.5 \mathrm{~Hz}, J 6.7 \mathrm{~Hz}), 2.31(1 \mathrm{H}, \mathrm{dd}, J 8.5 \mathrm{~Hz}, J 6.7 \mathrm{~Hz}), 3.86$ $(1 \mathrm{H}, \mathrm{d}, J 11.1 \mathrm{~Hz}), 4.03-4.20\left(2 \mathrm{H}, \mathrm{m}, \mathrm{CO}_{2} \mathrm{CH}_{2} \mathrm{CH}_{3}\right), 4.32(1 \mathrm{H}, \mathrm{d}, J 11.1 \mathrm{~Hz}), 9.52(1 \mathrm{H}, \mathrm{s}$, CHO) cis: $\delta 0.02$ (3 H, s, SiMe), $0.05(3 \mathrm{H}, \mathrm{s}, \mathrm{SiMe}) ; 0.84\left(9 \mathrm{H}, \mathrm{s}, \mathrm{SiCMe}_{3}\right), 1.23-1.28(3 \mathrm{H}$, m, $\left.\mathrm{CO}_{2} \mathrm{CH}_{2} \mathrm{CH}_{3}\right), 1.63(1 \mathrm{H}$, dd, $J 4.8 \mathrm{~Hz}, J 8.2 \mathrm{~Hz}), 1.91(1 \mathrm{H}, \mathrm{dd}, J 4.8 \mathrm{~Hz}, J 6.7 \mathrm{~Hz}), 2.33$ $(1 \mathrm{H}, \mathrm{dd}, J 8.2 \mathrm{~Hz}, J 6.7 \mathrm{~Hz}), 3.93(2 \mathrm{H}, \mathrm{s}), 4.03-4.20\left(2 \mathrm{H}, \mathrm{m}, \mathrm{CO}_{2} \mathrm{CH}_{2} \mathrm{CH}_{3}\right), 9.38(1 \mathrm{H}, \mathrm{s}$, $\mathrm{CHO}) ;{ }^{13} \mathrm{C}$ NMR $\left(67.5 \mathrm{MHz}, \mathrm{CDCl}_{3}\right)$ trans: $\delta-5.5\left(\mathrm{CH}_{3}\right),-5.4\left(\mathrm{CH}_{3}\right), 14.3\left(\mathrm{CH}_{3}\right), 18.3(\mathrm{C})$, $18.9(\mathrm{CH}), 25.8\left(\mathrm{CH}_{3}\right), 26.4(\mathrm{CH}), 40.6(\mathrm{C}), 59.4\left(\mathrm{CH}_{2}\right), 61.3\left(\mathrm{CH}_{2}\right), 170.1(\mathrm{C}), 200.2(\mathrm{C})$ cis: $\delta$-5.5 $\left(\mathrm{CH}_{3}\right),-5.4\left(\mathrm{CH}_{3}\right), 14.3\left(\mathrm{CH}_{3}\right), 16.1\left(\mathrm{CH}_{2}\right), 18.3(\mathrm{C}), 25.2(\mathrm{CH}), 25.8\left(\mathrm{CH}_{3}\right), 40.3(\mathrm{C})$, $59.1\left(\mathrm{CH}_{2}\right), 61.5\left(\mathrm{CH}_{2}\right), 171.0(\mathrm{C}), 200.4(\mathrm{C}) ; \mathrm{m} / z(\mathrm{CI}) 287\left(\mathrm{MH}^{+}\right)$[HRMS (CI) $\left[\mathrm{MH}^{+}\right.$] $\mathrm{C}_{14} \mathrm{H}_{27} \mathrm{O}_{4} \mathrm{Si}$ requires 287.1679. Found: 287.1681 (0.2 ppm error).

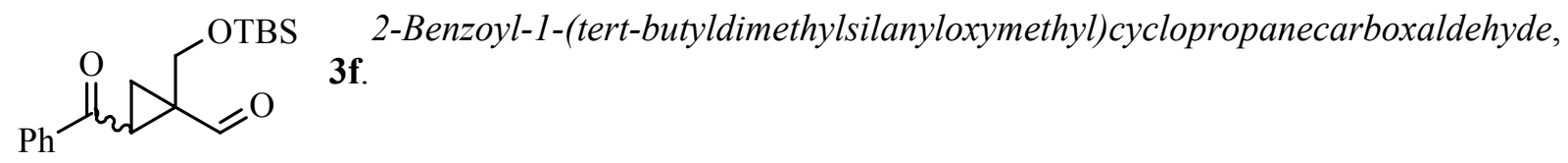

To a solution of allylic alcohol 1c $(51 \mathrm{mg}, 0.25 \mathrm{mmol})$ in dichloromethane $(5 \mathrm{~mL})$ was added powdered $4 \AA$ molecular sieves $(0.25 \mathrm{~g})$, (benzoylmethylene)dimethylsulfurane $\mathbf{2 b}$ (54 $\mathrm{mg}$, $0.30 \mathrm{mmol})$ and manganese dioxide $(217 \mathrm{mg}, 2.5 \mathrm{mmol})$. The mixture was heated at reflux for 4.5 hours then cooled to room temperature. The crude mixture was then filtered through celite $^{\circledR}$ and the residue washed with dichloromethane. After removal of the solvent, the residue was purified by flash column chromatography (19:1 to $9: 1 \mathrm{PE}:$ EtOAc) to give the title compound $\mathbf{3 f}$ (58 mg, 73\%) as a colorless oil, 2.0:1 trans:cis by ${ }^{1} \mathrm{H}$ NMR integration. $v_{\max }$ (film) 2954, 2929, 2857, 1716, 1675, 1451, 1255, 1223, $1090 \mathrm{~cm}^{-1} ;{ }^{1} \mathrm{H}$ NMR (400 MHz, $\left.\mathrm{CDCl}_{3}\right)$ trans: $\delta-0.29(3 \mathrm{H}, \mathrm{s}, \mathrm{SiMe}),-0.09(3 \mathrm{H}, \mathrm{s}, \mathrm{SiMe}) ; 0.62\left(9 \mathrm{H}, \mathrm{s}, \mathrm{SiCMe}_{3}\right), 1.49(1 \mathrm{H}$, dd, $J 4.1 \mathrm{~Hz}, J 7.9 \mathrm{~Hz}), 1.91(1 \mathrm{H}$, dd, $J 4.1 \mathrm{~Hz}, J 6.7 \mathrm{~Hz}), 3.35(1 \mathrm{H}$, dd, $J 7.9 \mathrm{~Hz}, J 6.7 \mathrm{~Hz})$, $3.58(1 \mathrm{H}, \mathrm{d}, J 11.3 \mathrm{~Hz}, \mathrm{OCHa}), 4.35(1 \mathrm{H}, \mathrm{d}, J 11.3 \mathrm{~Hz}, \mathrm{OCHb}), 7.39-7.49$ (2 H, m, arom.), 7.50-7.62 (2 H, m, arom.), $7.94(1 \mathrm{H}, \mathrm{d}, J 7.3 \mathrm{~Hz}$, arom. $), 9.76(1 \mathrm{H}, \mathrm{s}, \mathrm{CHO})$ cis: $\delta 0.11(3 \mathrm{H}$, s, SiMe), 0.12 (3 H, s, SiMe); $0.92\left(9 \mathrm{H}, \mathrm{s}, \mathrm{SiCMe}_{3}\right), 1.68(1 \mathrm{H}, \mathrm{dd}, J 4.6 \mathrm{~Hz}, J 7.6 \mathrm{~Hz}), 2.27$ $(1 \mathrm{H}, \mathrm{dd}, J 4.6 \mathrm{~Hz}, J 6.7 \mathrm{~Hz}), 3.35(1 \mathrm{H}, \mathrm{dd}, J 7.6 \mathrm{~Hz}, J 6.7 \mathrm{~Hz}), 3.79(1 \mathrm{H}, \mathrm{d}, J 10.8 \mathrm{~Hz}$, OCHa), $4.30(1 \mathrm{H}, \mathrm{d}, J 10.8 \mathrm{~Hz}, \mathrm{OCHb}), 7.39-7.49(2 \mathrm{H}, \mathrm{m}$, arom.), 7.50-7.62 (2 H, m, arom.), 8.03 (1 H, d, J 7.6 Hz, arom.), $9.12(1 \mathrm{H}, \mathrm{s}, \mathrm{CHO}) ;{ }^{13} \mathrm{C} \mathrm{NMR}\left(100 \mathrm{MHz}, \mathrm{CDCl}_{3}\right)$ trans: $\delta$-6.0 $\left(\mathrm{CH}_{3}\right),-5.8\left(\mathrm{CH}_{3}\right), 18.1(\mathrm{C}), 18.6\left(\mathrm{CH}_{2}\right), 25.6\left(\mathrm{CH}_{3}\right), 30.2(\mathrm{CH}), 43.7(\mathrm{C}), 59.2$ $\left(\mathrm{CH}_{2}\right), 128.5(\mathrm{CH}), 128.6(\mathrm{CH}), 133.2(\mathrm{CH}), 137.7(\mathrm{C}), 194.9(\mathrm{C}), 200.7(\mathrm{C})$ cis: $\delta-5.4$ $\left(\mathrm{CH}_{3}\right),-5.3\left(\mathrm{CH}_{3}\right), 16.6\left(\mathrm{CH}_{2}\right), 18.4(\mathrm{C}), 25.9\left(\mathrm{CH}_{3}\right), 30.1(\mathrm{CH}), 42.8(\mathrm{C}), 60.3\left(\mathrm{CH}_{2}\right), 128.6$ $(\mathrm{CH}), 128.8(\mathrm{CH}), 133.6(\mathrm{CH}), 137.3(\mathrm{C}), 195.9(\mathrm{C}), 199.4(\mathrm{C}) ; \mathrm{m} / z(\mathrm{CI}) 319\left(\mathrm{MH}^{+}\right)$[HRMS (CI) $\left[\mathrm{MH}^{+}\right] \mathrm{C}_{18} \mathrm{H}_{27} \mathrm{O}_{3} \mathrm{Si}$ requires 319.1729. Found: 319.1730 (0.1 ppm error).

$\mathrm{EtO}_{2} \mathrm{C}$ Diethyl 2,2'-carbonyldicyclopropanecarboxylate, $\mathbf{3 j}$.

To a solution of alcohol 1f $(50 \mu \mathrm{L}, 0.51 \mathrm{mmol})$ in dichloromethane $(10 \mathrm{~mL})$ was added powdered $4 \AA$ molecular sieves $(0.50 \mathrm{~g})$, (carbethoxymethylene)dimethylsulfurane 2a (166 $\mathrm{mg}, 1.12 \mathrm{mmol}$ ) and manganese dioxide $(443 \mathrm{mg}, 5.1 \mathrm{mmol})$. The solution was heated at reflux for 4 hours. The crude mixture was cooled and filtered through celite ${ }^{\circledR}$ and the residue washed with dichloromethane $(50 \mathrm{~mL})$. After removal of the solvent, the crude material was purified by flash column chromatography (5:1 PE:EtOAc) to give the title compound $\mathbf{3 j}$ ( 78 
mg, 60\%) as a colourless wax: $v_{\max }\left(\right.$ film) 1186, 1334, 1369, 1692, 1731, $2983 \mathrm{~cm}^{-1} ;{ }^{1} \mathrm{H}$ NMR $\left(270 \mathrm{MHz}, \mathrm{CDCl}_{3}\right) \delta 1.26(6 \mathrm{H}, \mathrm{t}, J 7.0 \mathrm{~Hz}), 1.40-1.46(4 \mathrm{H}, \mathrm{m}), 2.16-2.23(2 \mathrm{H}, \mathrm{m}), 2.58-2.65$ $(2 \mathrm{H}, \mathrm{m}), 4.14(4 \mathrm{H}, \mathrm{q}, J 7.0 \mathrm{~Hz}) ;{ }^{13} \mathrm{C} \mathrm{NMR}\left(67.5 \mathrm{MHz}, \mathrm{CDCl}_{3}\right) \delta 14.3\left(\mathrm{CH}_{3}\right), 17.8(\mathrm{CH}), 24.9$ (CH), $29.9\left(\mathrm{CH}_{2}\right), 61.3\left(\mathrm{CH}_{2}\right), 171.9(\mathrm{C}), 204.7(\mathrm{C}) ; \mathrm{m} / z(\mathrm{CI}) 255\left(\mathrm{MH}^{+}\right) 272\left(\mathrm{MNH}_{4}{ }^{+}\right)$[HRMS (CI) $\left[\mathrm{MNH}_{4}^{+}\right] \mathrm{C}_{13} \mathrm{H}_{22} \mathrm{NO}_{5}$ requires 272.1498. Found: 272.1500 (0.7 ppm error).

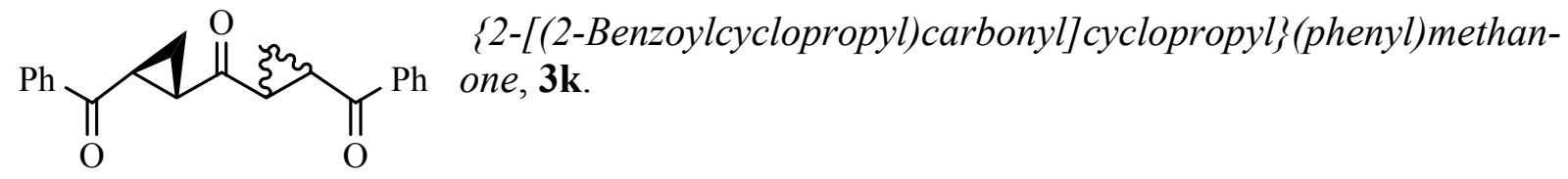

To a solution of alcohol 1f $(49 \mu \mathrm{L}, 0.50 \mathrm{mmol})$ in dichloromethane $(5 \mathrm{~mL})$ was added powdered $4 \AA$ molecular sieves $(0.50 \mathrm{~g})$, (benzoylmethylene)dimethylsulfurane $\mathbf{2 b}$ (216 mg, $1.20 \mathrm{mmol}$ ) and manganese dioxide (435 $\mathrm{mg}, 5.0 \mathrm{mmol}$ ). The solution was heated at reflux for 2.5 hours. The crude mixture was cooled and filtered through celite ${ }^{\circledR}$ and the residue washed with dichloromethane $(50 \mathrm{~mL})$. After removal of the solvent, the crude material was purified by flash column chromatography (19:1 to $4: 1 \mathrm{PE}:$ EtOAc) to give the title compound $\mathbf{3 k}$ (110 mg, 69\%) as a colourless solid: m.p. $148-150{ }^{\circ} \mathrm{C}$; $v_{\max }$ (film) 1660, 1349, $1225 \mathrm{~cm}^{-1} ;{ }^{1} \mathrm{H}$ NMR $\left(400 \mathrm{MHz}, \mathrm{CDCl}_{3}\right) \delta$ 1.58-1.78 (4 H, m), 2.84-2.97 (2 H, m), 3.23-3.38 (2 H, m), 7.38-7.51 (4 $\mathrm{H}, \mathrm{m}$, arom.), 7.52-7.64 (2 H, m, arom.), 7.91-8.06 (2 H, m, arom.); ${ }^{13} \mathrm{C} \mathrm{NMR}(100 \mathrm{MHz}$, $\left.\mathrm{CDCl}_{3}\right) \delta 20.2 / 20.3\left(\mathrm{CH}_{2}\right), 28.4 / 28.5(\mathrm{CH}), 32.3 / 32.4(\mathrm{CH}), 128.3 / 128.4(\mathrm{CH}), 128.7 / 128.8$ (CH), 133.4/133.5 (CH), 136.9 (C), 196.9 (C), 205.8 (C); m/z (CI) $319\left(\mathrm{MH}^{+}\right) 336\left(\mathrm{MNH}_{4}{ }^{+}\right)$ [HRMS (CI) $[\mathrm{MH}+] \mathrm{C}_{21} \mathrm{H}_{19} \mathrm{O}_{3}$ requires 319.1334. Found: 319.1330 (1.3 ppm error).
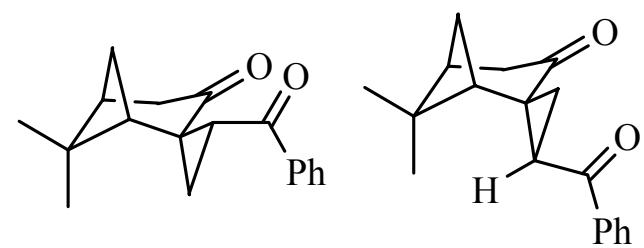

(1R,3R,6S,8S)-1-Benzoyl-7,7-dimethyl-6,8-methylenespiro[2.5]octan-4-one and $(1 S, 3 S, 6 S, 8 S)$-isomer, $3 \mathbf{3}$.

To a solution of allylic alcohol $1 \mathrm{~g}(76 \mathrm{mg}, 0.50 \mathrm{mmol})$ in dichloromethane $(5 \mathrm{~mL})$ was added powdered $4 \AA$ molecular sieves $(0.50 \mathrm{~g})$, (benzoylmethylene)dimethylsulfurane $2 \mathbf{b}$ (108 $\mathrm{mg}$, $0.60 \mathrm{mmol}$ ) and manganese dioxide (435 $\mathrm{mg}, 5.0 \mathrm{mmol}$ ). The mixture was heated at reflux for 16 hours then cooled to room temperature. The crude mixture was then filtered through celite $^{\circledR}$ and the residue washed with dichloromethane. After removal of the solvent, the residue was purified by flash column chromatography $(19: 1$ to $4: 1 \mathrm{PE}:$ EtOAc) togive the title compound 31 (102 mg, 76\%) as a colorless oil, 5.0:1 mixture of isomers by ${ }^{1} \mathrm{H}$ NMR integration. $v_{\max }$ (film) 2924, 1703, 1664, 1597, 1449, 1385, 1326, 1289, 1220, $1033 \mathrm{~cm}^{-1} ;{ }^{1} \mathrm{H}$ NMR (400 MHz, $\left.\mathrm{CDCl}_{3}\right)$ major: $\delta 0.94(3 \mathrm{H}, \mathrm{s}, \mathrm{Me}), 1.01(1 \mathrm{H}, \mathrm{d}, J 10.7 \mathrm{~Hz}) ; 1.28(3 \mathrm{H}, \mathrm{s}$, Me), $1.65(1 \mathrm{H}$, dd, $J 6.4 \mathrm{~Hz}, J 3.4 \mathrm{~Hz}), 1.72(1 \mathrm{H}$, dd, $J 7.9 \mathrm{~Hz}, J 3.4 \mathrm{~Hz}), 1.93(1 \mathrm{H}, \mathrm{t}, J 6.7$ Hz), 2.10-2.19 (1 H, m), 2.30-2.39 (1 H, m), $2.56(1 \mathrm{H}, \mathrm{dd}, J 19.2 \mathrm{~Hz}, J 2.8 \mathrm{~Hz}), 2.71(1 \mathrm{H}, \mathrm{dt}$, $J 19.2 \mathrm{~Hz}, J 2.5 \mathrm{~Hz}), 3.27(1 \mathrm{H}, \mathrm{t}, J 7.3 \mathrm{~Hz}), 7.39-7.45(2 \mathrm{H}, \mathrm{m}, \operatorname{arom}),. 7.49-7.55(2 \mathrm{H}, \mathrm{m}$, arom.), $7.91(1 \mathrm{H}, \mathrm{d}, J 7.6 \mathrm{~Hz}$, arom.) minor: $\delta 0.56(3 \mathrm{H}, \mathrm{s}, \mathrm{Me}), 1.13(3 \mathrm{H}, \mathrm{s}, \mathrm{Me}), 1.37(1 \mathrm{H}$, d, $J 10.5 \mathrm{~Hz}), 1.52-1.59(2 \mathrm{H}, \mathrm{m}), 1.93(1 \mathrm{H}$, obscured $), 2.10-2.19(2 \mathrm{H}, \mathrm{m}), 2.54(1 \mathrm{H}, \mathrm{dd}, J$ $19.3 \mathrm{~Hz}, J 2.8 \mathrm{~Hz}), 2.65(1 \mathrm{H}, \mathrm{dt}, J 19.3 \mathrm{~Hz}, J 2.8 \mathrm{~Hz}), 3.56(1 \mathrm{H}, \mathrm{t}, J 7.3 \mathrm{~Hz}), 7.39-7.45(2 \mathrm{H}$, $\mathrm{m}$, arom.), 7.49-7.55 (2 H, m, arom.), $7.93\left(1 \mathrm{H}, \mathrm{d}, J 7.3 \mathrm{~Hz}\right.$, arom.); ${ }^{13} \mathrm{C}$ NMR (100 MHz, $\left.\mathrm{CDCl}_{3}\right)$ major: $\delta 21.1\left(\mathrm{CH}_{3}\right), 22.5\left(\mathrm{CH}_{2}\right), 26.2\left(\mathrm{CH}_{3}\right), 32.2(\mathrm{CH}), 32.6\left(\mathrm{CH}_{2}\right), 38.6(\mathrm{CH}), 40.3$ $(\mathrm{CH}), 40.4(\mathrm{C}), 43.5\left(\mathrm{CH}_{2}\right), 45.3(\mathrm{CH}), 128.2(\mathrm{CH}), 128.6(\mathrm{CH}), 133.2(\mathrm{CH}), 137.9(\mathrm{C}), 195.8$ (C), $210.5(\mathrm{C})$ minor: $\delta 21.4\left(\mathrm{CH}_{3}\right), 22.1\left(\mathrm{CH}_{2}\right), 25.9\left(\mathrm{CH}_{3}\right), 32.6\left(\mathrm{CH}_{2}\right), 33.4(\mathrm{CH}), 38.7$ $(\mathrm{CH}), 39.5(\mathrm{C}), 40.0(\mathrm{CH}), 43.4\left(\mathrm{CH}_{2}\right), 45.6(\mathrm{C}), 128.4(\mathrm{CH}), 128.6(\mathrm{CH}), 133.1(\mathrm{CH}), 137.8$ 
(C), $195.8(\mathrm{C}), 210.4(\mathrm{C}) ; \mathrm{m} / z(\mathrm{CI}) 269\left(\mathrm{MH}^{+}\right)$[HRMS (CI) $\left[\mathrm{MH}^{+}\right] \mathrm{C}_{18} \mathrm{H}_{21} \mathrm{O}_{2}$ requires 269.1542. Found: 269.1537 (1.7 ppm error).

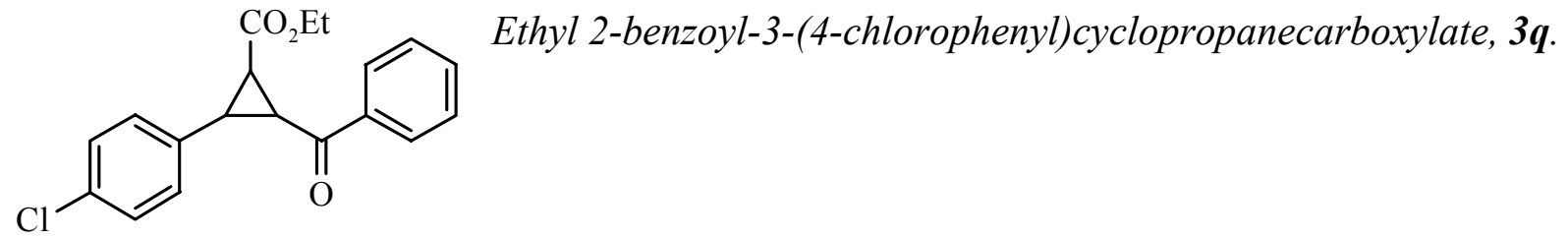

To a solution of alcohol $\mathbf{1 j}(113 \mathrm{mg}, 0.46 \mathrm{mmol})$ in $\mathrm{CH}_{2} \mathrm{Cl}_{2}(10 \mathrm{~mL})$ was added $4 \AA$ molecular sieves $(0.48 \mathrm{~g})$, (carbethoxymethylene)dimethylsulfurane $\mathbf{2 a}(85 \mathrm{mg}, 0.57 \mathrm{mmol})$ and manganese dioxide ( $435 \mathrm{mg}, 5 \mathrm{mmol}$ ). The mixture was heated at reflux for 14 hours. The crude mixture was filtered through celite ${ }^{\circledR}$ and the residue was washed with dichloromethane $(50 \mathrm{~mL})$ giving a pale yellow solution. After removal of the solvent, the crude material was purified by flash column chromatography (10:1 PE:EtOAc) to yield the title compound $\mathbf{3 q}$ (116 mg, 80\%) as a mixture of isomers, partially separable by chromatography. The first eluting material was shown to be A-3q, a colorless oil; $v_{\max }$ (film) 1014, 1191, 1372, 1450, 1494, 1680, 1730, $2980 \mathrm{~cm}^{-1} ;{ }^{1} \mathrm{H}$ NMR $\left(270 \mathrm{MHz}, \mathrm{CDCl}_{3}\right) \delta 1.11(3 \mathrm{H}, \mathrm{t}, J 7.2 \mathrm{~Hz}$, $\left.\mathrm{CO}_{2} \mathrm{CH}_{2} \mathrm{CH}_{3}\right), 2.82(1 \mathrm{H}, \mathrm{dd}, J 4.8 \mathrm{~Hz}, J 10.0 \mathrm{~Hz}), 3.21(1 \mathrm{H}, \mathrm{dd}, J 6.3 \mathrm{~Hz}, J 10.0 \mathrm{~Hz}), 3.80(1$ $\mathrm{H}, \mathrm{dd}, J 4.8 \mathrm{~Hz}, J 6.3 \mathrm{~Hz}), 4.01\left(2 \mathrm{H}, \mathrm{q}, J 7.2 \mathrm{~Hz}, \mathrm{CO}_{2} \mathrm{CH}_{2}\right), 7.27$ (4 H, br s, chlorophenyl H's), 7.48-7.56 (2 H, m, ArCO meta-H's), 7.61-7.67 (1 H, m, ArCO para-H), $8.10(2 \mathrm{H}, \mathrm{d}, J 7.2 \mathrm{~Hz}$, ArCO ortho-H's); ${ }^{13} \mathrm{C}$ NMR (67.5 MHz, $\left.\mathrm{CDCl}_{3}\right) \delta 14.3,29.8,32.3,34.3,61.3,128.6,128.7$, $130.4,132.2,133.3,133.4,133.8,137.0,168.8,196.5$. The next eluting material was an amorphous, colorless solid ; m.p. 102-105 ${ }^{\circ} \mathrm{C}$, shown to be a mixture of compounds B-3q and C-3q, 3.6:1 C:B (by ${ }^{1} \mathrm{H}$ NMR integration); $v_{\max }$ (film) 1093, 1185, 1372, 1453, 1496, 1680, 1729, $2977 \mathrm{~cm}^{-1}$; ${ }^{1} \mathrm{H}$ NMR $\left(270 \mathrm{MHz}, \mathrm{CDCl}_{3}\right) \mathbf{B - 3 q}: 1.30\left(3 \mathrm{H}, \mathrm{t}, J 7.2 \mathrm{~Hz}, \mathrm{CO}_{2} \mathrm{CH}_{2} \mathrm{CH}_{3}\right)$, $3.15(1 \mathrm{H}, \mathrm{dd}, J 5.2 \mathrm{~Hz}, J 6.3 \mathrm{~Hz}), 3.26(1 \mathrm{H}, \mathrm{dd}, J 6.3 \mathrm{~Hz}, J 10.4 \mathrm{~Hz}), 3.54(1 \mathrm{H}, \mathrm{dd}, J 4.8 \mathrm{~Hz}$, $J 10.0 \mathrm{~Hz}), 4.19\left(2 \mathrm{H}, \mathrm{q}, J 7.2 \mathrm{~Hz}, \mathrm{CO}_{2} \mathrm{CH}_{2}\right), 7.09-7.17(2 \mathrm{H}, \mathrm{m}, \mathrm{ArCl} \mathrm{H}$ 's), 7.23-7.33 (2 H, m, ArCl H's), 7.40-7.49 (2 H, m, ArCO meta-H's), 7.50-7.60 (1 H, m, ArCO ortho-H's), 7.90 (2 $\mathrm{H}, \mathrm{d}, J 8.4 \mathrm{~Hz}$, ArCO ortho-H's). C-3q: $1.10\left(3 \mathrm{H}, \mathrm{t}, J 7.2 \mathrm{~Hz}, \mathrm{CO}_{2} \mathrm{CH}_{2} \mathrm{CH}_{3}\right), 2.58(1 \mathrm{H}, \mathrm{dd}, J$ $5.9 \mathrm{~Hz}, J 9.7 \mathrm{~Hz}), 3.02(1 \mathrm{H}, \mathrm{dd}, J 6.3 \mathrm{~Hz}, J 9.7 \mathrm{~Hz}), 3.31(1 \mathrm{H}, \mathrm{t}, J 6.1 \mathrm{~Hz}), 4.04(2 \mathrm{H}, \mathrm{q}, J 7.2$ $\mathrm{Hz}, \mathrm{CO}_{2} \mathrm{CH}_{2}$ ), 7.09-7.17 (2 H, m, ArCl H's), 7.23-7.33 (2 H, m, ArCl H's), 7.40-7.49 (2 H, m, ArCO meta-H's), 7.50-7.60 (1 H, m, ArCO ortho-H's), $7.98(2 \mathrm{H}, \mathrm{d}, J 8.1 \mathrm{~Hz}$, ArCO orthoH's); ${ }^{13} \mathrm{C}$ NMR (67.5 MHz, $\left.\mathrm{CDCl}_{3}\right) \delta$ C-3q: $14.3\left(\mathrm{CH}_{3}\right), 29.0(\mathrm{CH}), 31.7(\mathrm{CH}), 34.1(\mathrm{CH})$, $61.3\left(\mathrm{CH}_{2}\right), 128.1(\mathrm{CH}), 128.5(\mathrm{CH}), 128.8(\mathrm{CH}), 129.0(\mathrm{CH}), 133.0(\mathrm{C}), 133.5(\mathrm{CH}), 136.7$ (C), 136.9 (C), 169.0 (C), 193.5 (C); m/z (CI) $328\left(\mathrm{MH}^{+}\right)$[HRMS (CI) $\left[\mathrm{MH}^{+}\right] \mathrm{C}_{19} \mathrm{H}_{18} \mathrm{O}_{3}{ }^{35} \mathrm{Cl}$ requires 329.0944. Found 329.0947 (0.8 ppm error).

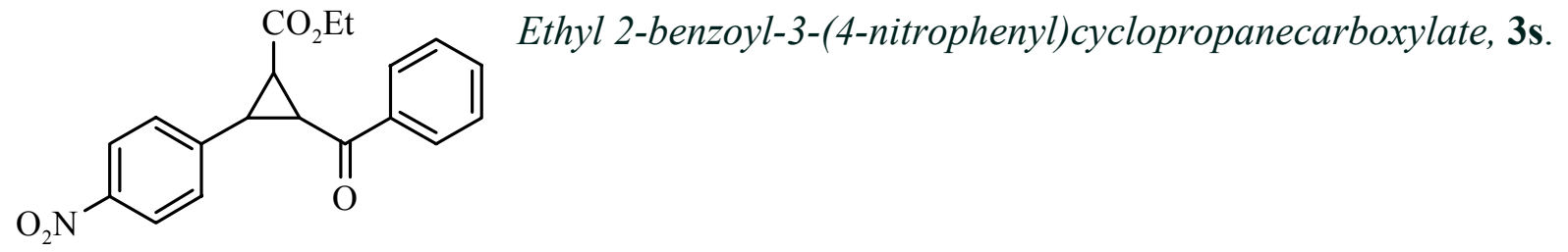

To a solution of alcohol $1 \mathrm{n}(71 \mathrm{mg}, 0.28 \mathrm{mmol})$ in THF $(10 \mathrm{~mL})$ was added $4 \AA$ molecular sieves $(0.30 \mathrm{~g})$, (carbethoxymethylene)dimethylsulfurane $\mathbf{2 a}(82 \mathrm{mg}, 0.56 \mathrm{mmol})$ and manganese dioxide $(242 \mathrm{mg}, 2.78 \mathrm{mmol})$. The mixture was heated at reflux for 14 hours. The crude mixture was filtered through celite ${ }^{\circledR}$ and the residue was washed with dichloromethane $(50 \mathrm{~mL})$ giving a pale yellow solution. After removal of the solvent, the 
crude material was purified by flash column chromatography (5:1 PE:EtOAc) to yield the title compound 3s ( $85 \mathrm{mg}, 90 \%)$ as a mixture of isomers, partially separable by chromatography. The first eluting material was shown to be compound $\mathbf{A - 3 s}$, a pale yellow oil ; $v_{\max }$ (film) 1010, 1344, 1515, 1597, 2852, 2923, $3390 \mathrm{~cm}^{-1} ;{ }^{1} \mathrm{H}$ NMR $\left(270 \mathrm{MHz}, \mathrm{CDCl}_{3}\right) \delta 1.12(3 \mathrm{H}, \mathrm{t}, J$ $\left.7.1 \mathrm{~Hz}, \mathrm{CO}_{2} \mathrm{CH}_{2} \mathrm{CH}_{3}\right), 2.88(1 \mathrm{H}$, dd, $J 4.8 \mathrm{~Hz}, J 10.0 \mathrm{~Hz}), 3.32(1 \mathrm{H}$, dd, $J 6.3 \mathrm{~Hz}, J 9.7 \mathrm{~Hz})$, $3.88(1 \mathrm{H}, \mathrm{dd}, J 4.8 \mathrm{~Hz}, J 6.3 \mathrm{~Hz}), 4.02\left(2 \mathrm{H}, \mathrm{q}, J 7.1 \mathrm{~Hz}, \mathrm{CO}_{2} \mathrm{CH}_{2}\right), 7.45-7.58(4 \mathrm{H}, \mathrm{m}), 7.60-$ $7.69(1 \mathrm{H}, \mathrm{m}), 8.07-8.12(2 \mathrm{H}, \mathrm{m}), 8.13-8.20(2 \mathrm{H}, \mathrm{m}) ;{ }^{13} \mathrm{C} \mathrm{NMR}\left(67.5 \mathrm{MHz}, \mathrm{CDCl}_{3}\right) \delta 14.2$ $\left(\mathrm{CH}_{3}\right), 29.9(\mathrm{CH}), 32.4(\mathrm{CH}), 33.9(\mathrm{CH}), 61.5\left(\mathrm{CH}_{2}\right), 123.5(\mathrm{CH}), 128.5(\mathrm{CH}), 129.0(\mathrm{CH})$, $130.0(\mathrm{CH}), 134.0(\mathrm{CH}), 136.7(\mathrm{C}), 142.5(\mathrm{C}), 147.3(\mathrm{C}), 168.5(\mathrm{C}), 195.9(\mathrm{C}) ; \mathrm{m} / z(\mathrm{CI}) 240$ $\left(\mathrm{MH}^{+}\right) 257\left(\mathrm{MNH}_{4}{ }^{+}\right)$[HRMS (CI) $\left[\mathrm{MNH}_{4}{ }^{+}\right] \mathrm{C}_{19} \mathrm{H}_{21} \mathrm{~N}_{2} \mathrm{O}_{5}$ requires 357.1450. Found: 357.1448 (0.2 ppm error). The next eluting material was a pale yellow oil, shown to be compounds B-3s and C-3s, 1.6:1 C:B (by ${ }^{1} \mathrm{H}$ NMR integration); $v_{\max }$ (film) 1184, 1347, 1520, 1601, 1679, 1730, $2983 \mathrm{~cm}^{-1}$; ${ }^{1} \mathrm{H}$ NMR $\left(270 \mathrm{MHz}, \mathrm{CDCl}_{3}\right) \delta$ B-3s: $1.34\left(3 \mathrm{H}, \mathrm{t}, J 7.0 \mathrm{~Hz}, \mathrm{CO}_{2} \mathrm{CH}_{2} \mathrm{CH}_{3}\right), 3.25(1$ $\mathrm{H}, \mathrm{dd}, J 5.0 \mathrm{~Hz}, J 6.3 \mathrm{~Hz}), 3.38(1 \mathrm{H}$, dd, J $10.0 \mathrm{~Hz}, J 6.3 \mathrm{~Hz}), 3.66(1 \mathrm{H}$, dd, J $5.0 \mathrm{~Hz}, J 10.0$ Hz), $4.24(2 \mathrm{H}, \mathrm{q}, J 7.0 \mathrm{~Hz}), 7.34-7.43(2 \mathrm{H}, \mathrm{m}), 7.44-7.53(2 \mathrm{H}, \mathrm{m}), 7.53-7.64$ (1 H, m), 7.89$7.96(2 \mathrm{H}, \mathrm{m}), 7.99-8.10(2 \mathrm{H}, \mathrm{m}) \mathbf{C}-3 \mathrm{~s}: 1.12\left(3 \mathrm{H}, \mathrm{t}, J 7.4 \mathrm{~Hz}, \mathrm{CO}_{2} \mathrm{CH}_{2} \mathrm{CH}_{3}\right), 2.72(1 \mathrm{H}, \mathrm{dd}, J$ $5.9 \mathrm{~Hz}, J 9.7 \mathrm{~Hz}), 3.16(1 \mathrm{H}, \mathrm{dd}, J 6.3 \mathrm{~Hz}, J 9.7 \mathrm{~Hz}), 3.46(1 \mathrm{H}, \mathrm{dd}, J 5.9 \mathrm{~Hz}, J 6.3 \mathrm{~Hz}), 4.07$ $\left(2 \mathrm{H}, \mathrm{q}, J=7.4 \mathrm{~Hz}, \mathrm{CO}_{2} \mathrm{CH}_{2}\right), 7.34-7.43(2 \mathrm{H}, \mathrm{m}), 7.44-7.53(2 \mathrm{H}, \mathrm{m}), 7.53-7.64(1 \mathrm{H}, \mathrm{m})$, 7.99-8.10 $(2 \mathrm{H}, \mathrm{m}), 8.17-8.23(2 \mathrm{H}, \mathrm{m}) ;{ }^{13} \mathrm{C} \mathrm{NMR}\left(67.5 \mathrm{MHz}, \mathrm{CDCl}_{3}\right) \delta$ (There was difficulty in assigning signals to a given isomer, but the following were observed) $14.1\left(\mathrm{CH}_{3}\right), 14.3$ $\left(\mathrm{CH}_{3}\right), 26.7(\mathrm{CH}), 28.9(\mathrm{CH}), 32.3(\mathrm{CH}), 34.9(\mathrm{CH}), 35.0(\mathrm{CH}), 35.6(\mathrm{CH}), 61.6\left(\mathrm{CH}_{2}\right), 61.8$ $\left(\mathrm{CH}_{2}\right), 123.5$ (ArC), 124.2 (ArC), 127.5 (ArC), 128.3 (ArC), 128.5 (ArC), 128.8 (ArC), 128.9 (ArC), 129.9 (ArC), 133.8 (ArC), 136.6 (C), 136.7 (C), 141.1 (C), 146.0 (C), 147.2 (C), 168.4 (C), 171.5 (C), $192.8(\mathrm{C}) ; \mathrm{m} / z$ (CI) $240\left(\mathrm{MH}^{+}\right)$[HRMS (CI) $\left[\mathrm{MH}^{+}\right] \mathrm{C}_{19} \mathrm{H}_{18} \mathrm{NO}_{5}$ requires 340.1184. Found : 340.1184 (0.0 ppm error). 


$$
1
$$




\section{2-Benzoyl-1-methylcyclopropanecarboxaldehyde, $\mathbf{3 d}$}

\section{Title: $k 3868$ sar}

Converted from "E: POSTDOC5IPD479_1|K3868S 1.GXD"
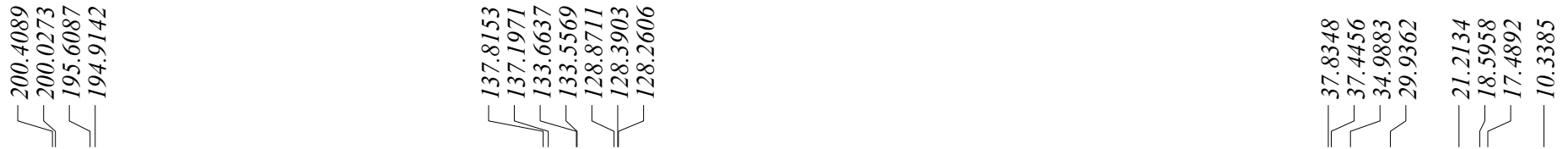

$\infty$

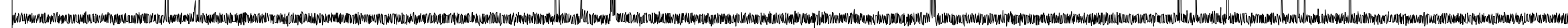


Ethyl 2-(tert-butyldimethylsilanyloxymethyl)-2-formyl-cyclopropane-carboxylate, $\mathbf{3 e}$

Title: b6882mfo

Converted from "N:|SAR113|MAGALIE $|M O 29| 1 H \mid B 6882 M F O . G X D "$

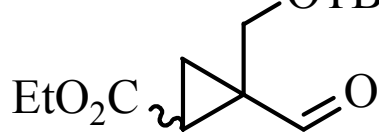

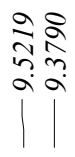

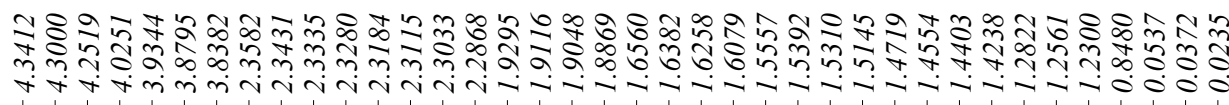

5 
Ethyl 2-(tert-butyldimethylsilanyloxymethyl)-2-formyl-cyclopropane-carboxylate, $\mathbf{3 e}$ Title: b6883mfo

Converted from "E:|NMRDAT 1|MO29|13C|B6883MFO.GXD"

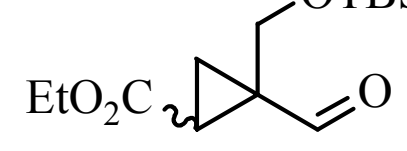

$\frac{n}{0}$

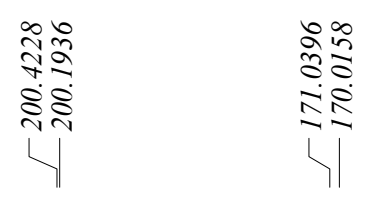
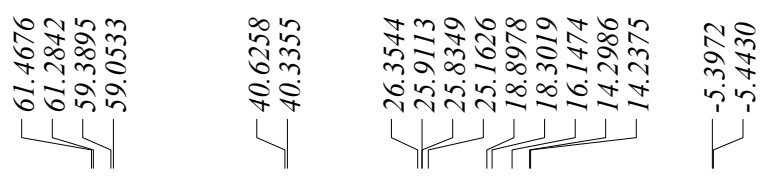

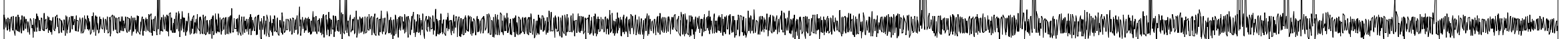

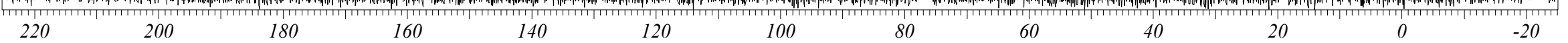
(ppm) 


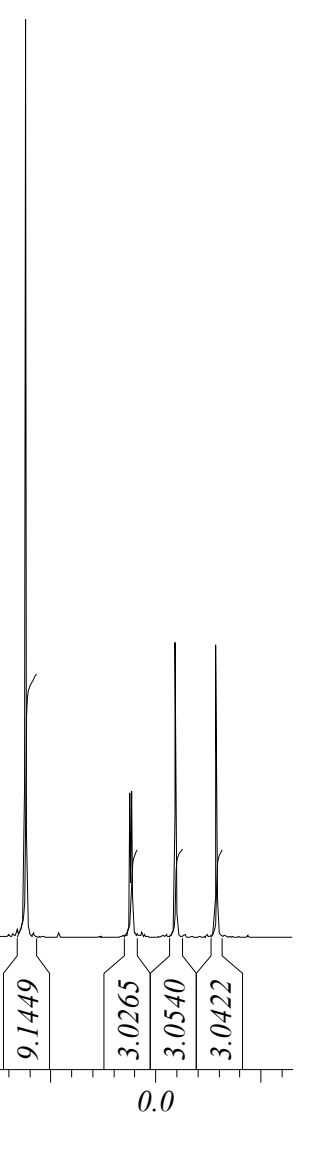




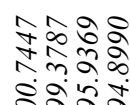

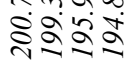

$\frac{\pi}{N}$

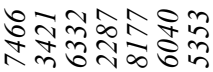



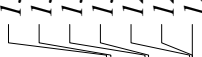

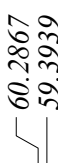

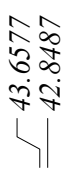

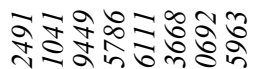

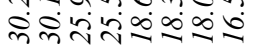

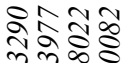

जि

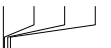




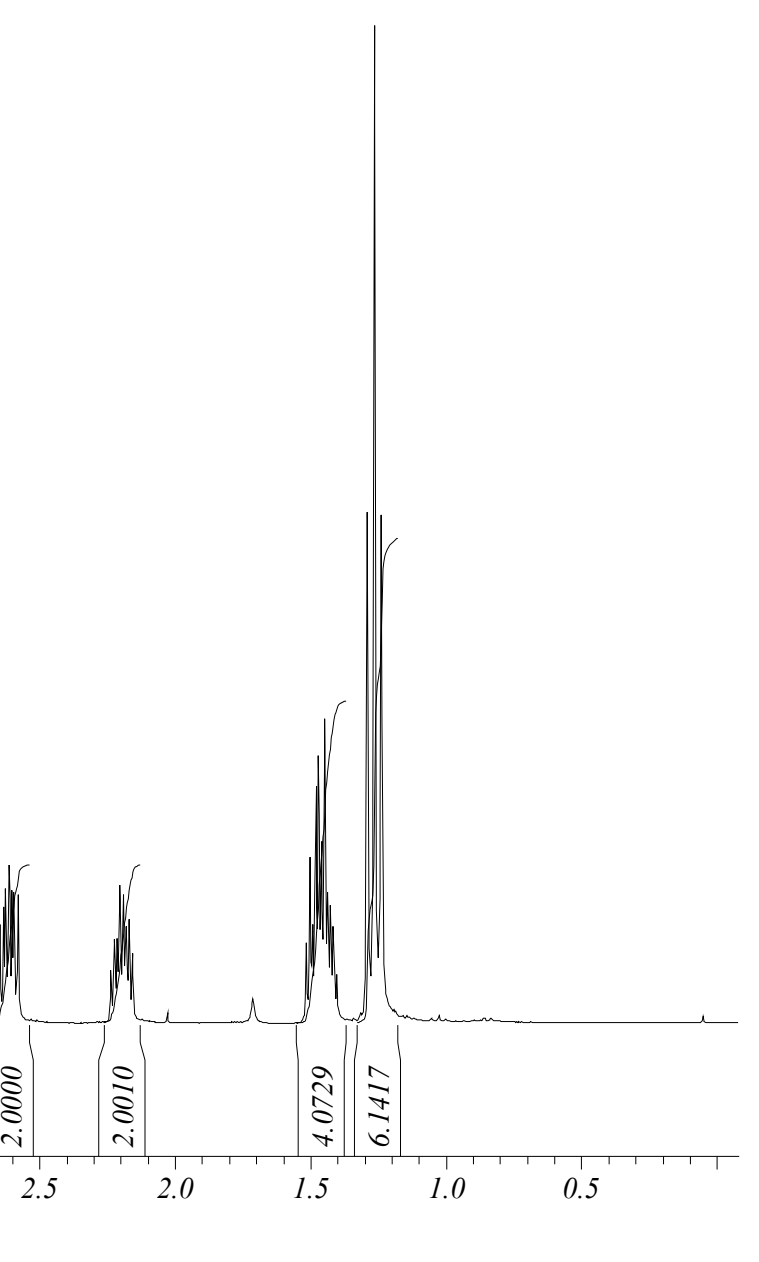



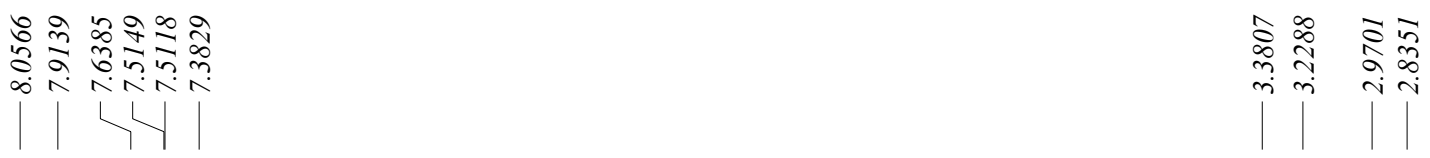

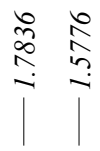
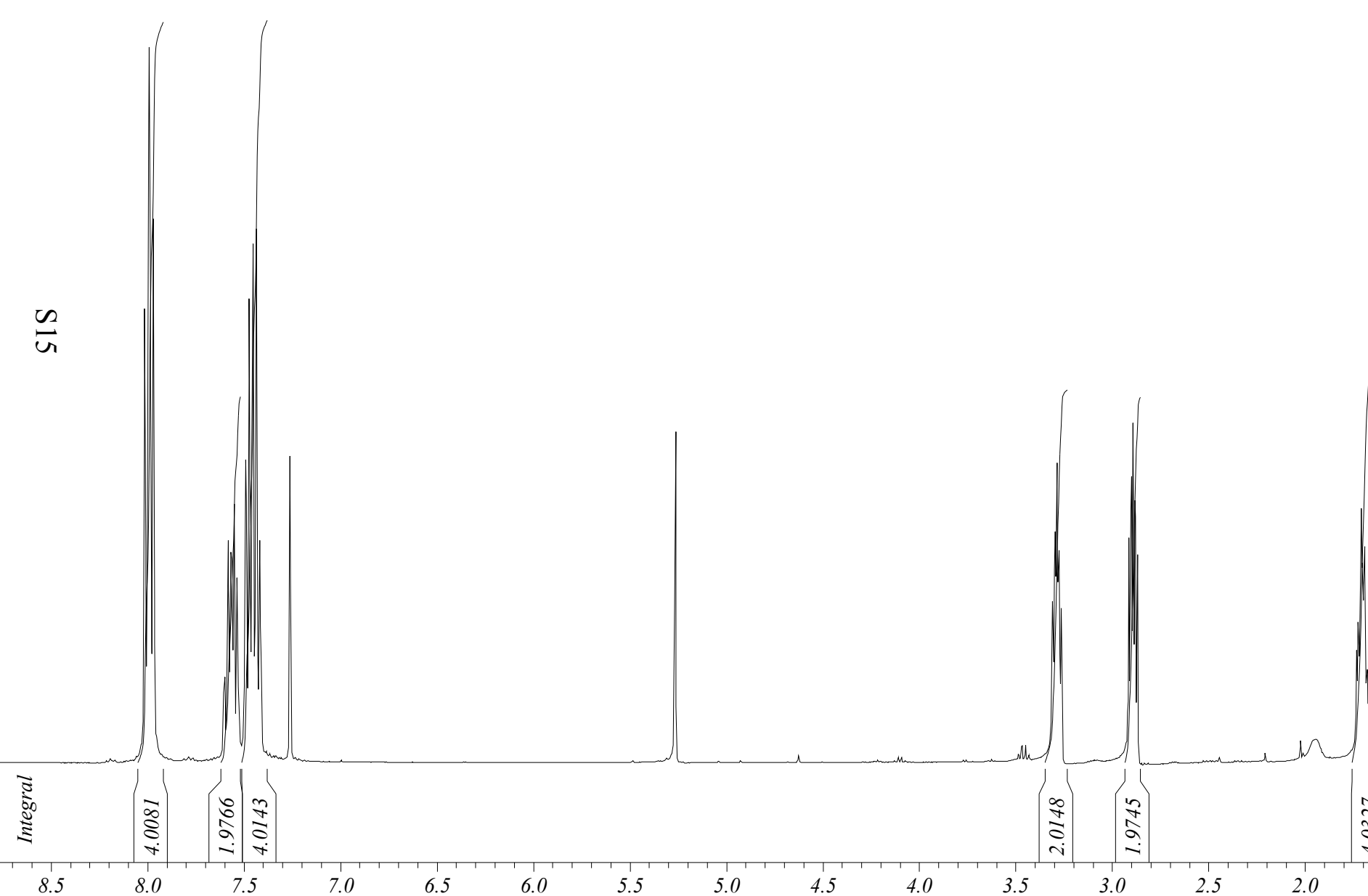


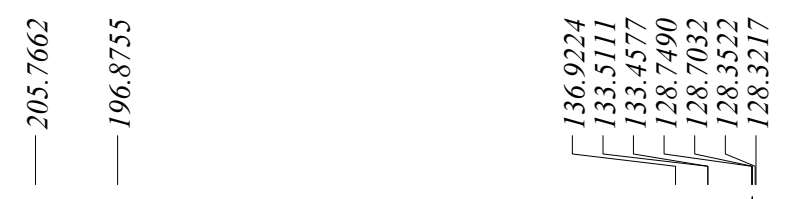

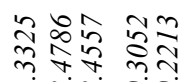

miल

$\frac{2}{a}$ 
Converted from "E:|NMRDAT I|MO23|MO23-1|1H|B6251MFO.GXD"

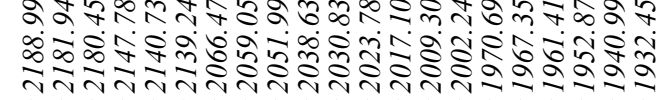

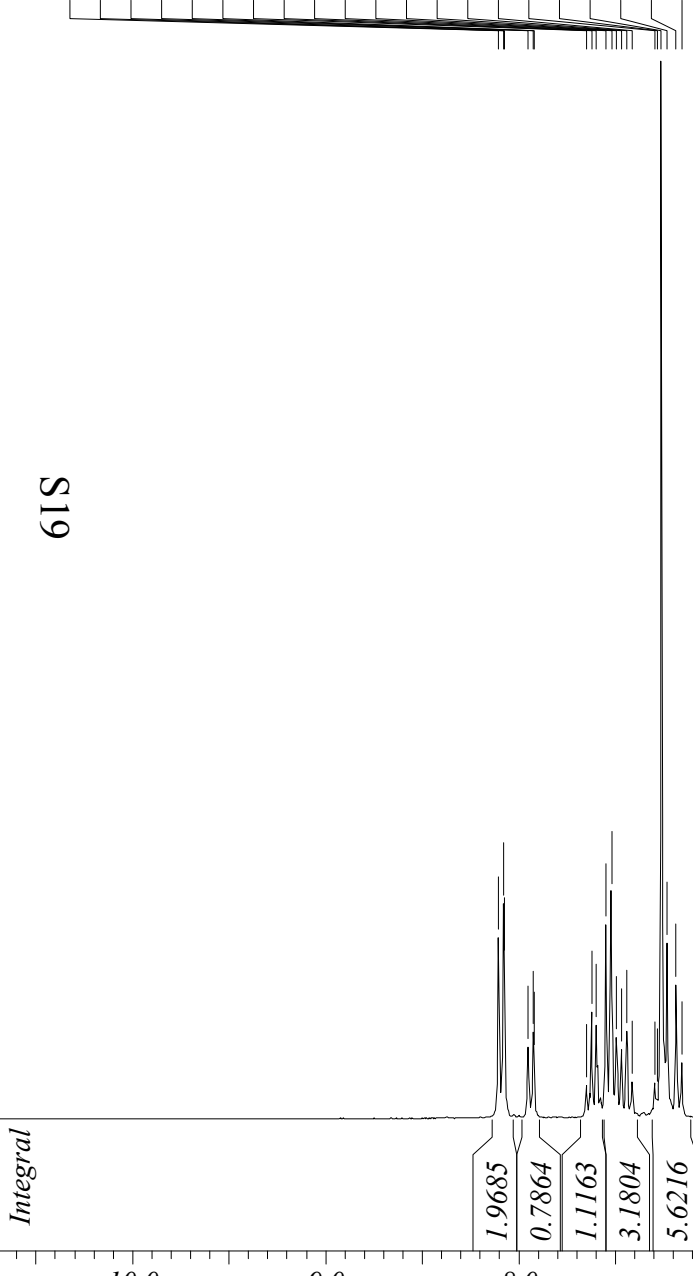

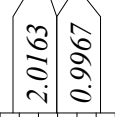

4.0

(ppm)
ชุส

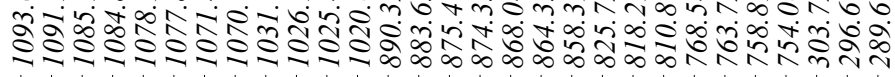

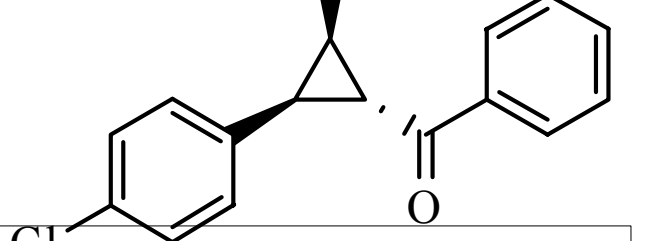

$\mathrm{Cl}$

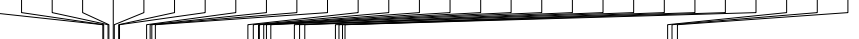

مे

1.0 


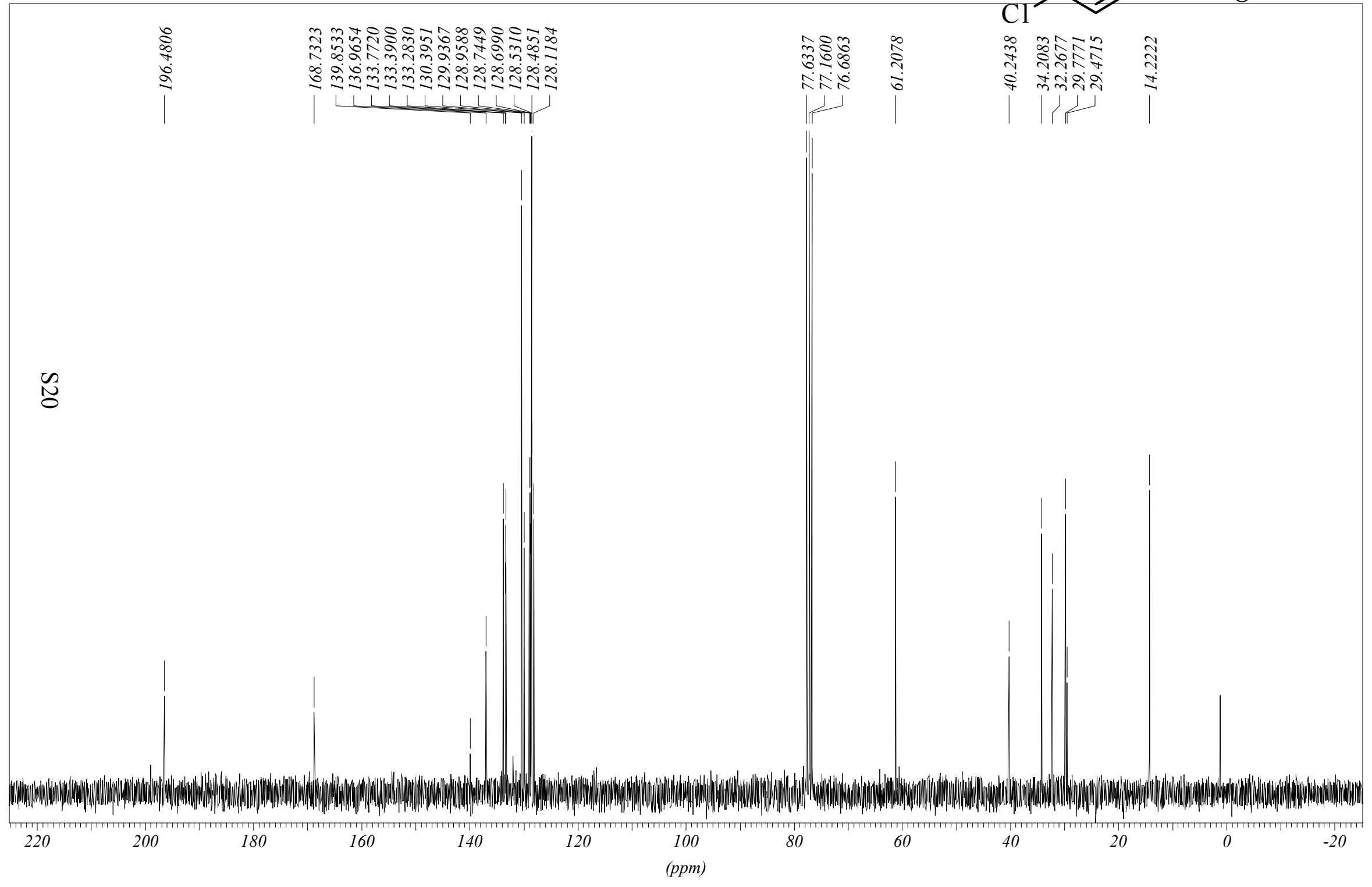




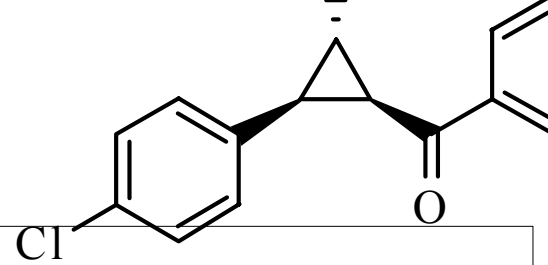

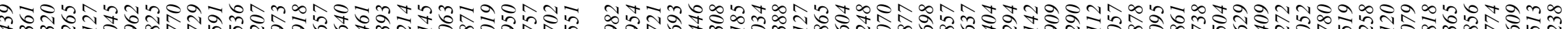

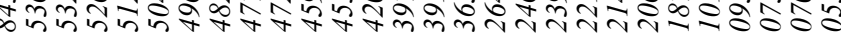

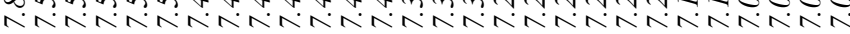

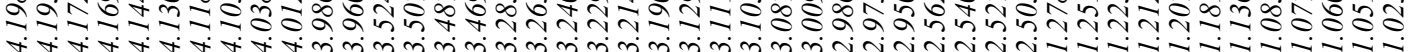

$\underset{N}{\sim}$

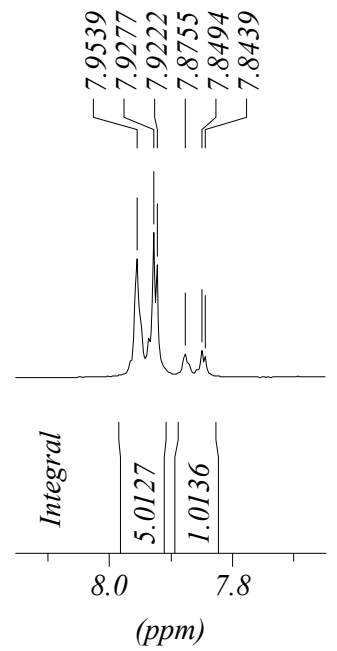

$\frac{\sqrt{2}}{3}$ |ा|ा|||||
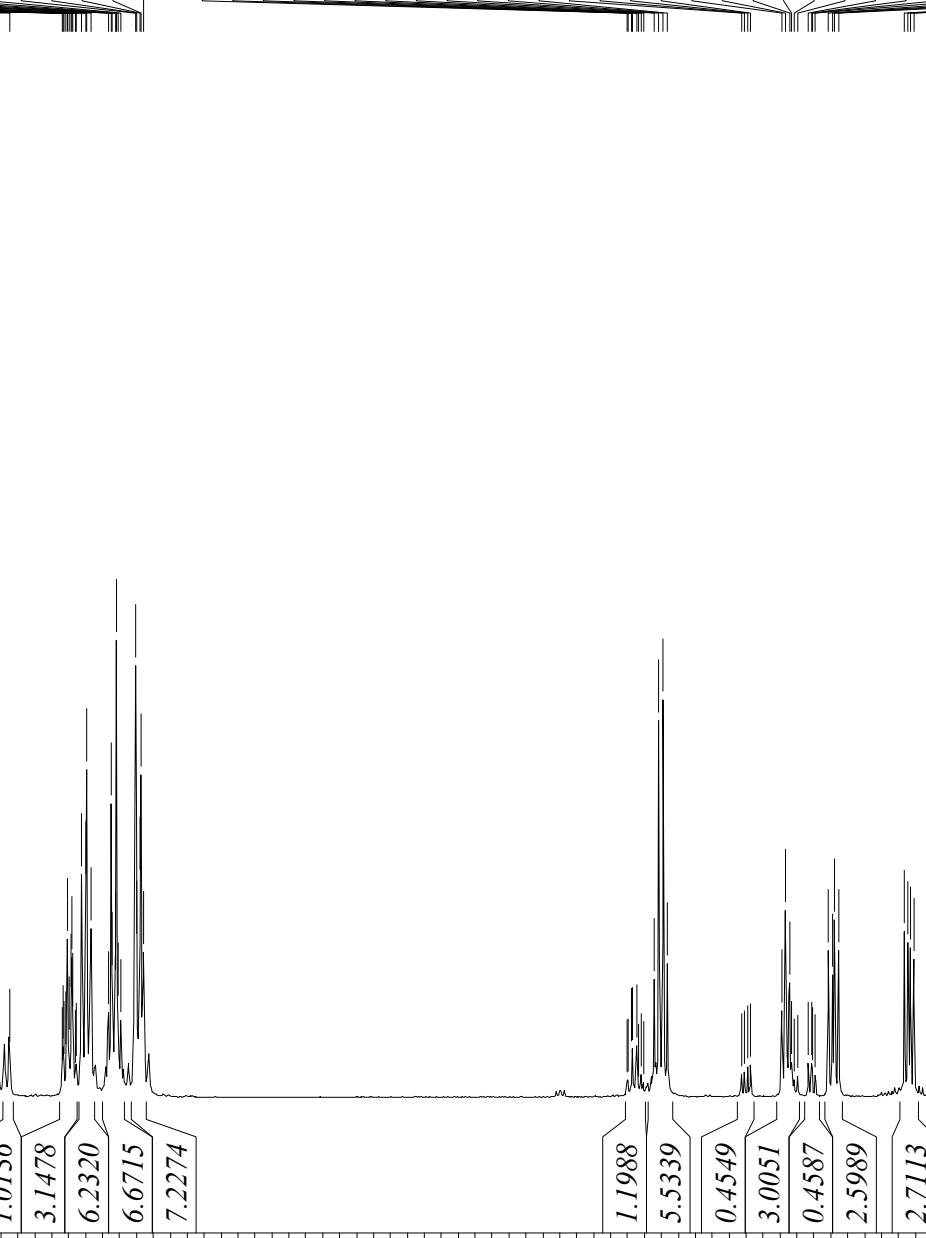
Title: $k 0395 \mathrm{mfo}$

Converted from " $E:|N M R D A T \sim 1| M O 23|M O 23-3| 13 C C O R \sim 1 \mid K 0395 M \sim 1 . G X D "$

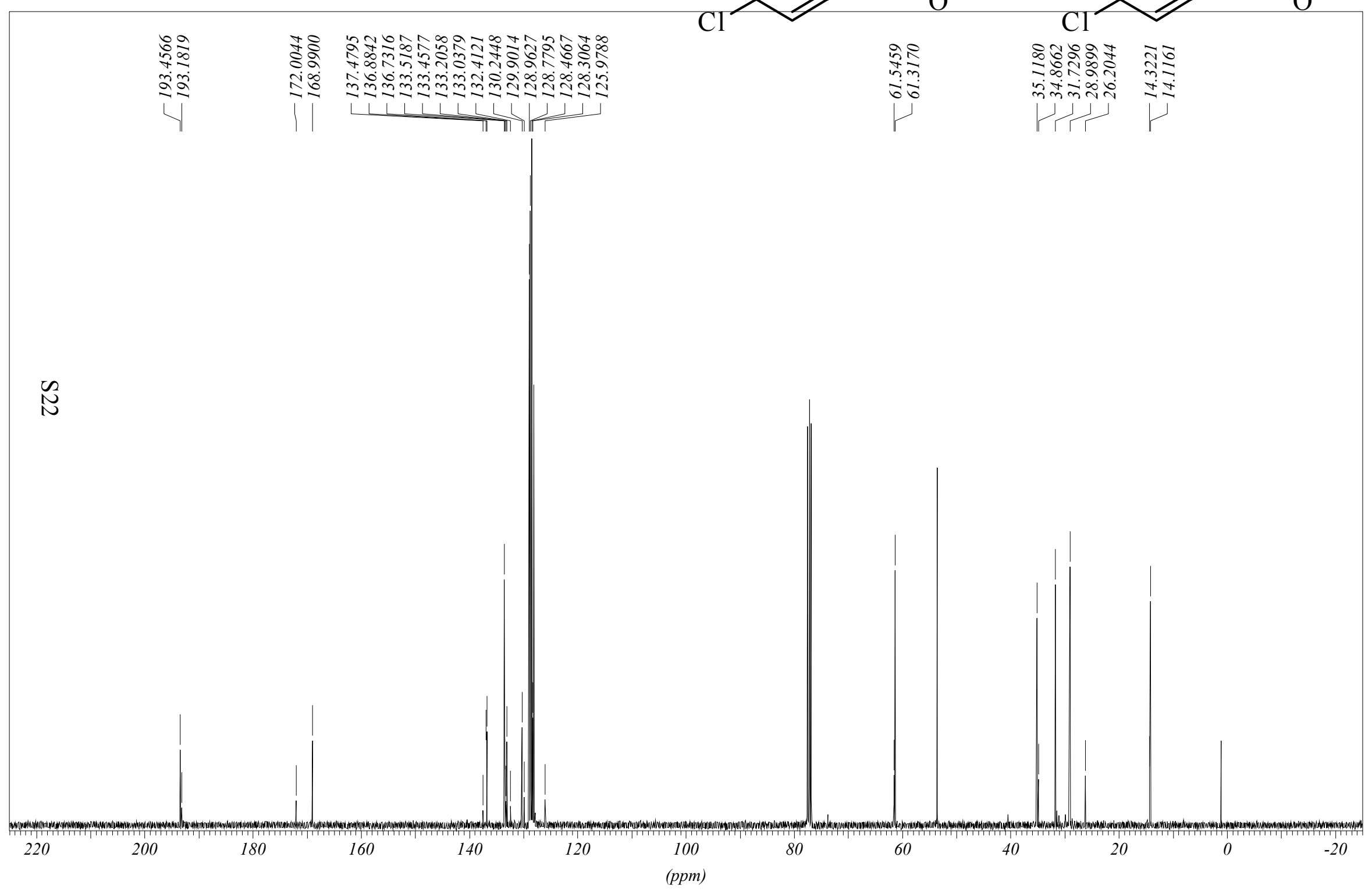




\section{$|N M R D A T \sim 1| M O 27 \backslash 3-6 \backslash 1 H \mid B 6586 M F O . G X D$}

讵 
$|N M R D A T \sim 1| M O 27|3-6 \backslash 13 C| B 6587 M F O . G X D$

\begin{tabular}{|c|c|c|}
\hline $\begin{array}{l}\overline{7} \\
2 \\
2 \\
2\end{array}$ & 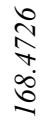 & 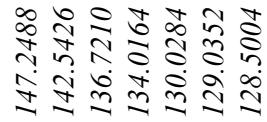 \\
\hline
\end{tabular}

$\tilde{+}$

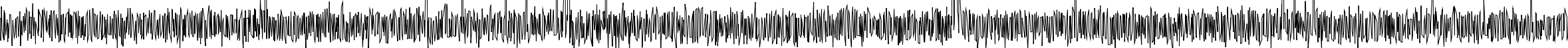


Converted from "E:|NMRDAT I|MO27|10-17|13C|B6589M 1.GXD"

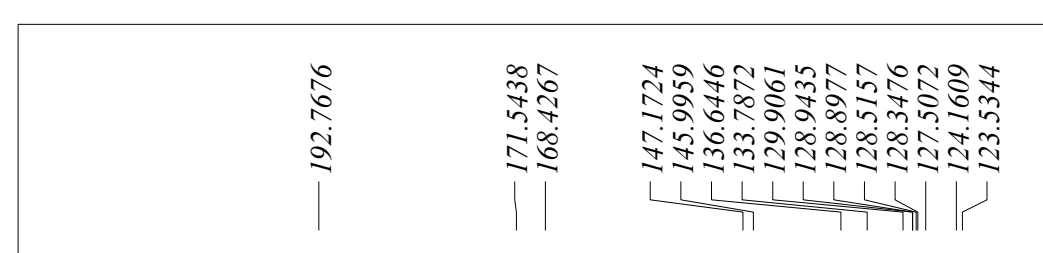

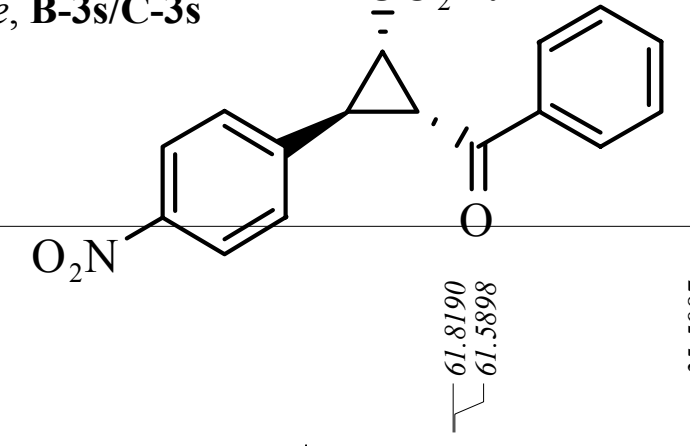

$\mathrm{O}_{2} \mathrm{~N}$

केंग

승ำ

mं்

N 